\title{
No More Than Three: \\ Technoeconomic Mixed Integer Nonlinear Programming (MINLP) Optimization of Mixed Suspension, Mixed Product Removal (MSMPR) Crystallizer Cascades for Melitracen, an Antidepressant API
}

Samir Diab, Dimitrios I. Gerogiorgis*

Institute for Materials and Processes (IMP), School of Engineering, University of Edinburgh, The Kings Buildings, Edinburgh, EH9 3FB, Scotland, UK

*Corresponding Author: D.Gerogiorgis@ed.ac.uk

\section{SUPPLEMENTARY INFORMATION}

Table S1 lists optimal mass flowrates of key flowsheet (Fig. 2 of main manuscript text) streams and crystallizer holdups for different considered plant API capacities $\left(Q_{\text {API }}\right)$ and number of implemented crystallizers $(N)$ presented in Fig. 12 of the main manuscript.

Table S1: Optimal mass flowrates of key flowsheet streams and crystallizer holdups for both considered plant capacities $\left(Q_{\mathrm{API}}\right)$ and different numbers of MSMPR crystallizers $(N)$. PR $=$ Product stream, $\mathrm{HD}_{i}=$ Holdup in MSMPR $i, \mathrm{R}_{i}=$ Recycle stream.

\begin{tabular}{|c|c|c|c|c|c|c|}
\hline \multicolumn{7}{|c|}{$Q_{\mathrm{API}}=10^{3} \mathrm{~kg} \mathrm{API} \mathrm{yr}^{-1}$} \\
\hline \multirow[b]{2}{*}{ Stream } & \multirow[b]{2}{*}{ Units } & \multicolumn{5}{|c|}{$N$} \\
\hline & & 1 & 2 & 3 & 4 & 5 \\
\hline$\overline{\mathrm{PR}}$ & $\mathrm{kg} \mathrm{hr}^{-1}$ & 0.125 & 0.125 & 0.125 & 0.125 & 0.125 \\
\hline $\mathrm{HD}_{1}$ & $\mathrm{~kg}$ & 0.546 & 0.248 & 0.107 & 0.087 & 0.067 \\
\hline $\mathrm{R}_{1}$ & $\mathrm{~kg} \mathrm{hr}^{-1}$ & 0.585 & 0.201 & 0.068 & 0.077 & 0.077 \\
\hline $\mathrm{HD}_{2}$ & $\mathrm{~kg}$ & $(-)$ & 0.273 & 0.110 & 0.092 & 0.070 \\
\hline $\mathrm{R}_{2}$ & $\mathrm{~kg} \mathrm{hr}^{-1}$ & $(-)$ & 0.201 & 0 & 0.077 & 0.077 \\
\hline $\mathrm{HD}_{3}$ & $\mathrm{~kg}$ & $(-)$ & $(-)$ & 0.131 & 0.096 & 0.073 \\
\hline $\mathrm{R}_{3}$ & $\mathrm{~kg} \mathrm{hr}^{-1}$ & $(-)$ & $(-)$ & 0.068 & 0 & 0.077 \\
\hline $\mathrm{HD}_{4}$ & $\mathrm{~kg}$ & $(-)$ & $(-)$ & $(-)$ & 0.111 & 0.082 \\
\hline $\mathrm{R}_{4}$ & $\mathrm{~kg} \mathrm{hr}^{-1}$ & $(-)$ & $(-)$ & $(-)$ & 0.077 & 0 \\
\hline $\mathrm{HD}_{5}$ & $\mathrm{~kg}$ & $(-)$ & $(-)$ & $(-)$ & $(-)$ & 0.089 \\
\hline $\mathrm{R}_{5}$ & $\mathrm{~kg} \mathrm{hr}^{-1}$ & $(-)$ & $(-)$ & $(-)$ & $(-)$ & 0.077 \\
\hline WS & $\mathrm{kg} \mathrm{hr}^{-1}$ & 0.118 & 0.073 & 0.031 & 0.035 & 0.041 \\
\hline \multicolumn{7}{|c|}{$Q_{\mathrm{API}}=10^{4} \mathrm{~kg} \mathrm{API} \mathrm{yr}^{-1}$} \\
\hline & & \multicolumn{5}{|c|}{$N$} \\
\hline Stream & Units & 1 & 2 & 3 & 4 & 5 \\
\hline$\overline{\mathrm{PR}}$ & $\mathrm{kg} \mathrm{hr}^{-1}$ & 1.250 & 1.250 & 1.250 & 1.250 & 1.250 \\
\hline $\mathrm{HD}_{1}$ & $\mathrm{~kg}$ & 1.778 & 0.806 & 0.347 & 0.283 & 0.218 \\
\hline $\mathrm{R}_{1}$ & $\mathrm{~kg} \mathrm{hr}^{-1}$ & 5.852 & 2.012 & 0.680 & 0.770 & 0.773 \\
\hline $\mathrm{HD}_{2}$ & $\mathrm{~kg}$ & $(-)$ & 0.889 & 0.358 & 0.229 & 0.228 \\
\hline $\mathrm{R}_{2}$ & $\mathrm{~kg} \mathrm{hr}^{-1}$ & $(-)$ & 2.012 & 0 & 0.770 & 0.773 \\
\hline $\mathrm{HD}_{3}$ & $\mathrm{~kg}$ & $(-)$ & $(-)$ & 0.426 & 0.312 & 0.238 \\
\hline $\mathrm{R}_{3}$ & $\mathrm{~kg} \mathrm{hr}^{-1}$ & $(-)$ & $(-)$ & 0.680 & 0 & 0.773 \\
\hline $\mathrm{HD}_{4}$ & $\mathrm{~kg}$ & $(-)$ & $(-)$ & $(-)$ & 0.365 & 0.270 \\
\hline $\mathrm{R}_{4}$ & $\mathrm{~kg} \mathrm{hr}^{-1}$ & $(-)$ & $(-)$ & $(-)$ & 0.770 & 0 \\
\hline $\mathrm{HD}_{5}$ & $\mathrm{~kg}$ & $(-)$ & $(-)$ & $(-)$ & $(-)$ & 0.294 \\
\hline $\mathrm{R}_{5}$ & $\mathrm{~kg} \mathrm{hr}^{-1}$ & $(-)$ & $(-)$ & $(-)$ & $(-)$ & 0.773 \\
\hline WS & $\mathrm{kg} \mathrm{hr}^{-1}$ & 1.268 & 0.794 & 0.339 & 0.367 & 0.426 \\
\hline
\end{tabular}


Table S2 provides a detailed list of cash flows for optimal plant designs (corresponding to data presented in Fig. S1 for both considered plant capacities $\left(Q_{\mathrm{API}}\right)$ and numbers of implemented crystallizers $(N)$ at the end of the plant lifetime.

Table S2: Cash flow for optimal plant designs $\left(10^{3} \mathrm{GBP}\right)$ for both considered plant capacities $\left(Q_{\mathrm{API}}\right)$ and different numbers of MSMPR crystallizers $(N)$.

\begin{tabular}{|c|c|c|c|c|c|c|c|}
\hline \multicolumn{8}{|c|}{${\underline{Q_{\mathrm{API}}}}=10^{3} \mathrm{~kg} \mathrm{API} \mathrm{yr}^{-1}$} \\
\hline & & \multirow[b]{2}{*}{ Stage, $i$} & \multicolumn{5}{|c|}{$N$} \\
\hline & & & 1 & 2 & 3 & 4 & 5 \\
\hline \multirow{6}{*}{$\overline{C a p E x}$} & $B L I C$ & 1 & -66.528 & -43.827 & -28.725 & -26.209 & -23.226 \\
\hline & & 2 & $(-)$ & -43.902 & -28.834 & -26.220 & -23.239 \\
\hline & & 3 & $(-)$ & $(-)$ & -29.373 & -26.220 & -23.252 \\
\hline & & 4 & $(-)$ & $(-)$ & $(-)$ & -26.244 & -23.252 \\
\hline & & 5 & $(-)$ & $(-)$ & $(-)$ & $(-)$ & -23.265 \\
\hline & $W C C$ & & -14.163 & -18.191 & -17.781 & -21.421 & -23.736 \\
\hline \multirow[t]{7}{*}{$O p E x$} & Utilities (Cooling) & 1 & -7.365 & -6.316 & -5.148 & -5.148 & -4.503 \\
\hline & & 2 & $(-)$ & -5.579 & -4.122 & -4.122 & -4.008 \\
\hline & & 3 & $(-)$ & $(-)$ & -2.834 & -2.834 & -2.726 \\
\hline & & 4 & $(-)$ & $(-)$ & $(-)$ & -3.882 & -3.392 \\
\hline & & 5 & $(-)$ & $(-)$ & $(-)$ & $(-)$ & -4.337 \\
\hline & Utilities (Materials) & & -300.511 & -232.274 & -148.591 & -167.285 & -186.042 \\
\hline & Waste & & -132.050 & -98.425 & -58.964 & -66.505 & -73.795 \\
\hline \multicolumn{2}{|l|}{ Profit } & & +623.111 & +623.111 & +623.111 & +623.111 & +623.111 \\
\hline \multicolumn{8}{|c|}{$Q_{\mathrm{API}}=10^{4} \mathrm{~kg} \mathrm{API} \mathrm{yr}^{-1}$} \\
\hline & & & \multicolumn{5}{|c|}{$N$} \\
\hline & & Stage, $i$ & 1 & 2 & 3 & 4 & 5 \\
\hline \multirow[t]{6}{*}{$\overline{C a p E x}$} & $B L I C$ & 1 & -126.361 & -82.760 & -53.819 & -49.293 & -43.779 \\
\hline & & 2 & $(-)$ & -82.902 & -54.026 & -49.316 & -43.803 \\
\hline & & 3 & $(-)$ & $(-)$ & -55.047 & -49.316 & -43.828 \\
\hline & & 4 & $(-)$ & $(-)$ & $(-)$ & -49.360 & -43.828 \\
\hline & & 5 & $(-)$ & $(-)$ & $(-)$ & $(-)$ & -43.853 \\
\hline & $W C C$ & & -34.048 & -39.724 & -36.615 & -43.927 & -48.754 \\
\hline \multirow[t]{7}{*}{$O p E x$} & Utilities (Cooling) & 1 & -75.945 & -65.291 & -53.415 & -53.415 & -46.857 \\
\hline & & 2 & $(-)$ & -57.473 & -42.677 & -42.677 & -41.519 \\
\hline & & 3 & $(-)$ & $(-)$ & -29.273 & -29.273 & -28.175 \\
\hline & & 4 & $(-)$ & $(-)$ & $(-)$ & -39.492 & -34.582 \\
\hline & & 5 & $(-)$ & $(-)$ & $(-)$ & $(-)$ & -43.963 \\
\hline & Utilities (Materials) & & $-2,999.882$ & $-2,253.239$ & $-1,379.772$ & $-1,528.100$ & $-1,687.371$ \\
\hline & Waste & & $-1,352.117$ & $-1,007.107$ & -603.493 & -672.032 & -745.629 \\
\hline Profit & & & $+6,231.105$ & $+6,231.105$ & $+6,231.105$ & $+6,231.105$ & $+6,231.105$ \\
\hline
\end{tabular}




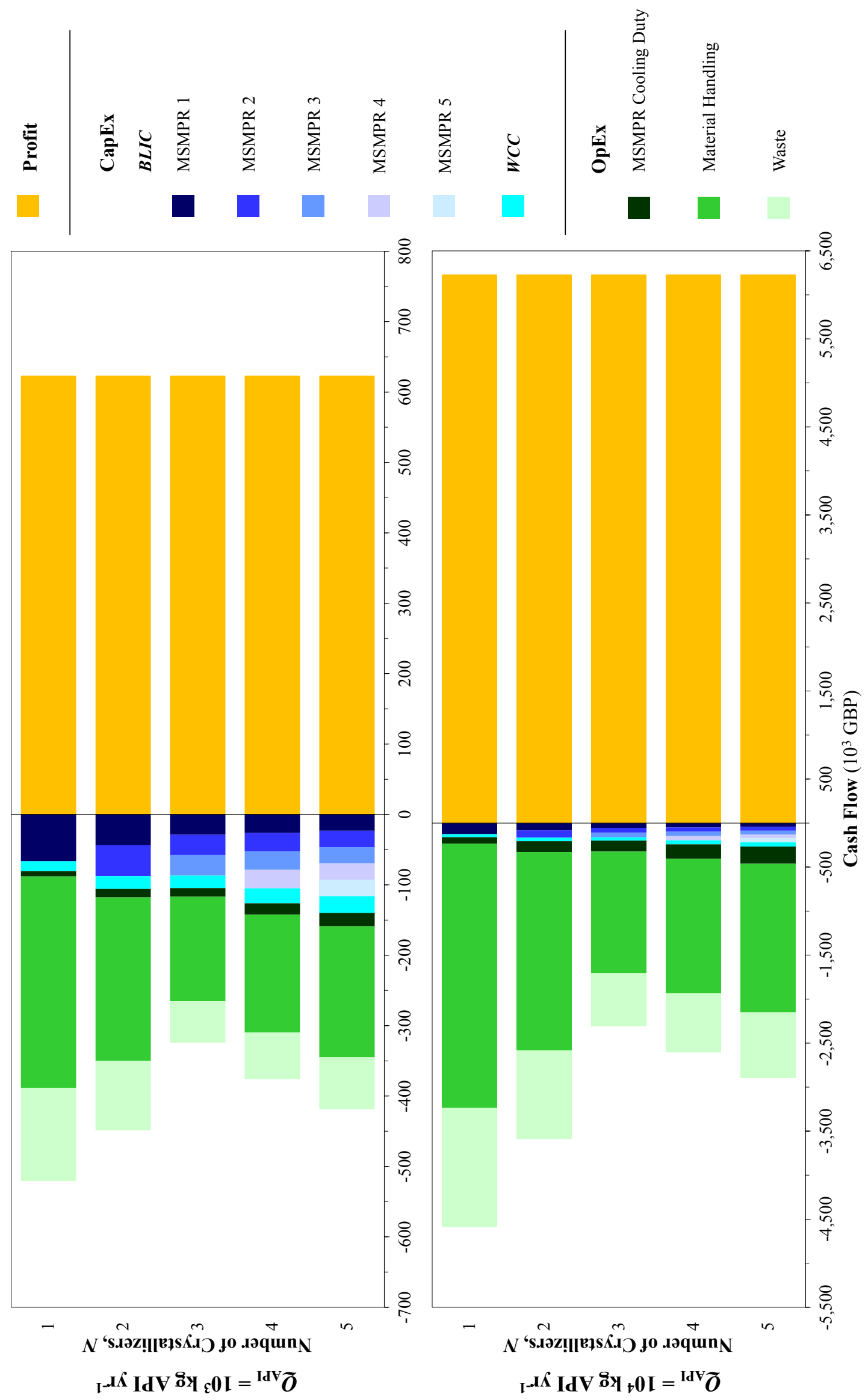

Figure S1: Optimal cash flows per unit crystallizer at the end of the plant lifetime for different capacities $\left(Q_{\mathrm{API}}\right)$ and numbers of crystallizers $(N)$. 